\title{
Subtrochanteric Fracture Treatment: A Retrospective Study of 46 Patients
}

\author{
Fan Li Weilin Sang Qiugen Wang Jianhua Huang Haimin Lu \\ Department of Orthopaedic Surgery, The First People's Hospital of Shanghai Jiaotong University, Shanghai, China
}

\section{Key Words}

Subtrochanteric fracture $\cdot$ Medial cortex $\cdot$ Nonunion

\begin{abstract}
Objective: To investigate whether or not reconstruction of the medial cortex in subtrochanteric fracture can reduce the risks of internal fixation failure, fracture malunion, and nonunion. Subjects and Methods: Between 2005 and 2008, 46 patients with subtrochanteric fracture were treated with intramedullary nailing and followed up. The average age was 42 years. According to the Seinsheimer classification, 26 patients were type IIIA, 7 were type IIIB, 11 were type IV and 2 were type $\mathrm{V}$. Medial cortex alignment was achieved under traction in 40 cases. In 5 cases, the medial fractures were too far away and we used minimally invasive open reduction with wire or titanium cable cerclage. In 1 case of type IIIA subtrochanteric fracture, the displaced medial fracture was not reconstructed and was fixed with a long Gamma nail. We reviewed all patients' radiographs before and after treatment to evaluate bone healing. Results: The 46 patients were followed up for an average of 24.3 months. Bone union was achieved within an average of 18.7 weeks in 45 cases. One case, a type IIIA fracture nonunion with displaced medial fracture, was not reconstructed. Instead, we used a free fibular transplantation on the medial side and fracture union was achieved 6 months after revision surgery. Conclusions:
\end{abstract}

In reconstructing the medial cortex in subtrochanteric fracture treatment, continuity and integrity are important. They can reduce the failure of internal fixation and the incidence of nonunion.

Copyright $\odot 2011$ S. Karger AG, Basel

\section{Introduction}

Subtrochanteric fractures are femoral fractures that occur below the lesser trochanter to $5 \mathrm{~cm}$ distal in the shaft of the femur. They account for $7-34 \%$ of femoral fractures [1-4]. Unlike the intertrochanteric region, the subtrochanteric region is full of hard, cortical bone, with a high level of stress and strong muscles that can cause fracture displacement [5]. Subtrochanteric fractures typically occur at the junction between the trabecular and cortical bones where the mechanical stress in the femur is greatest. Subtrochanteric fractures cause great difficulty in reduction and fixation, and treating them remains a challenge for orthopaedic surgeons.

There have been some reports of implant failure and nonunion $[6,7]$. Furthermore, even if the fracture heals, it can still affect the quality of life, especially in elderly patients [8]. Previous studies have typically focused on which implant provides better fixation to achieve stability and a high healing rate.

\section{KARGER}

Fax +4161306 1234

E-Mail karger@karger.ch

www.karger.com (c) 2011 S. Karger AG, Basel

1011-7571/11/0206-0519\$38.00/0

Accessible online at:

www.karger.com/mpp 
Restoring continuity and integrity of the medial cortex has not received great attention in the treatment of subtrochanteric fractures. We retrospectively analyzed 46 cases between 2005 and 2008 to discuss the clinical significance of restoration of the medial cortex in the treatment of subtrochanteric fractures.

\section{Subjects and Methods}

We retrospectively analyzed 46 cases of subtrochanteric fracture treated between 2005 and 2008. The fractures were found among 30 males and 16 females; their average age was 42 years. Of the 46 cases, 31 involved car accidents, 12 involved falls from high buildings, and 3 involved walking falls. According to the Seinsheimer classification [9], 26 cases were type IIIA, 7 were type IIIB, 11 were type IV, and 2 were type V. Four cases also involved ipsilateral tibia fractures, and 3 cases involved ipsilateral femoral shaft fractures. Two cases of Gustilo type IIIA subtrochanteric fracture were treated with debridement and external fixation in the emergency department, followed by intramedullary nailing 10 days later. All other cases were treated with intramedullary nailing at the first stage without bone grafting. We used proximal femoral nails in 25 cases, Gamma nails in 7 cases, and Trigen nails in 14 cases. Apparent medial cortex displacement was found in 6 cases. We used a minimally invasive open reduction with wire or titanium cable cerclage, except in 1 case.

Skin traction or femoral condyle bone traction was routinely done when patients were admitted to the hospital. Surgery was performed within 3 days, except for open fractures. General anaesthesia and a traction bed were used in operations. Closed reductions were attempted using clamps, bone hooks, and a spiked pusher. Then we sought to observe and estimate the reduction with a fluoroscopic image, especially of the medial cortex. If the medial cortex was comminuted (39 cases in this series), we did not open the fracture site and fixed it with intramedullary nailing using a closed method. If the medial fracture was not comminuted and apparently displaced, we used a minimally invasive open reduction and wire or titanium cable cerclage. We believe it is important to avoid excessive soft tissue stripping and to maintain the continuity of the subtrochanteric medial cortex.

\section{Results}

All patients were followed up for 14-47 months (mean 24.3 months). Bone union was achieved in 45 cases within 15-21 weeks of surgery (mean 18.7 weeks). According to the traumatic hip rating scale, functional results were excellent in 30 cases and good in 16.

There was 1 case of type IIIA fracture with displaced medial fracture that was not reconstructed; it was treated with closed reduction with long Gamma nail fixation at the first stage. Nonunion was found 7 months after the operation. We removed the distal locking screws for 12

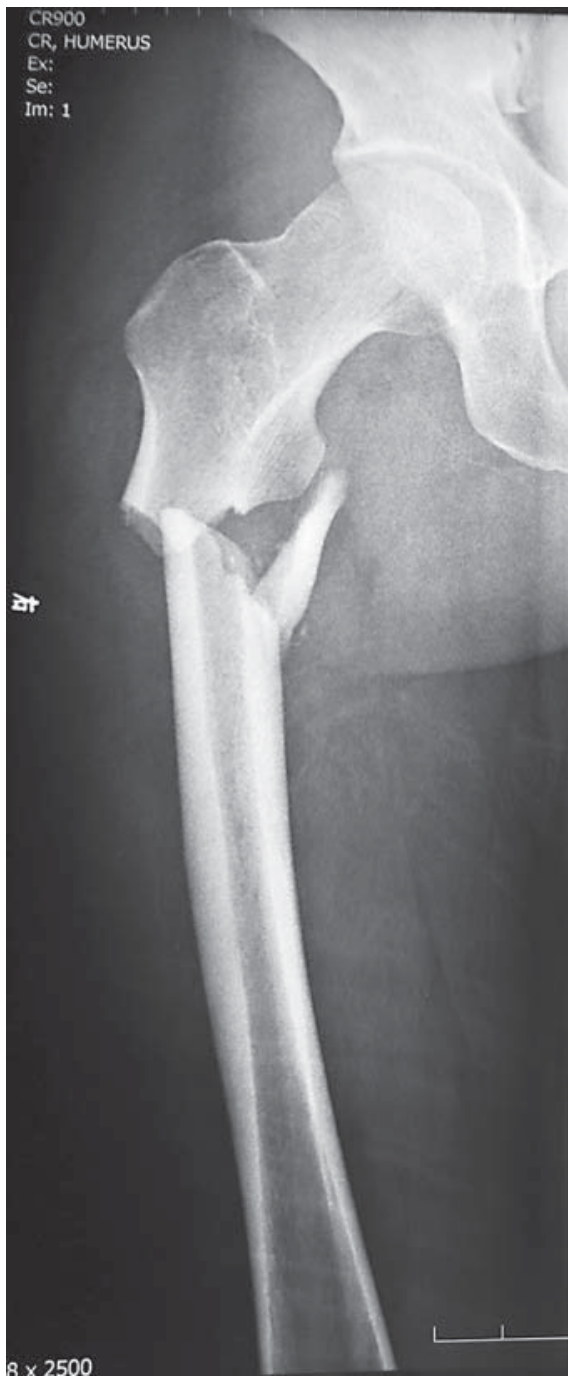

Fig. 1. A 34-year-old patient suffered from a traffic accident and got a subtrochanteric fracture.

months and locked again, but this still failed. Then we used a free fibular transplantation on the medial side and a locking plate on the anterior side 43 months after the first operation, and fracture union was achieved 6 months after this revision surgery (fig. 1-7).

\section{Discussion}

Subtrochanteric fracture, the most difficult of femur fractures to manage, typically occurs in two age groups. In young and healthy individuals, the injury typically results from high-energy trauma, whereas in the elderly 


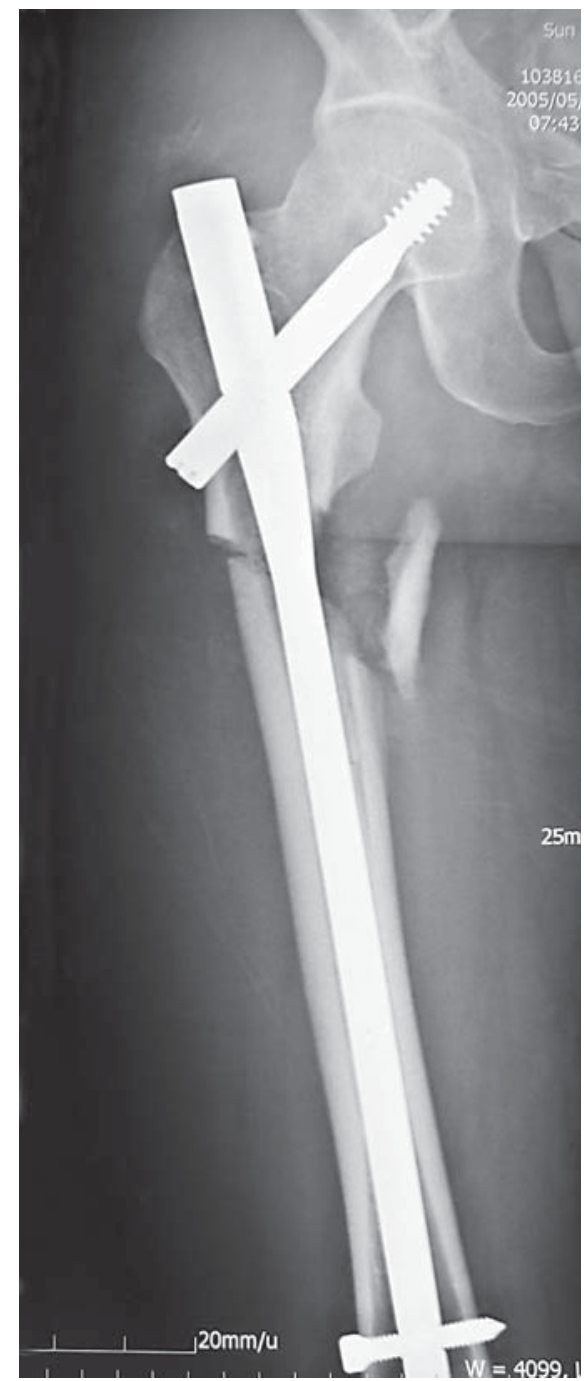

Fig. 2. Operation was done 3 days after the injury. A long Gamma nail was used to fix the fracture.

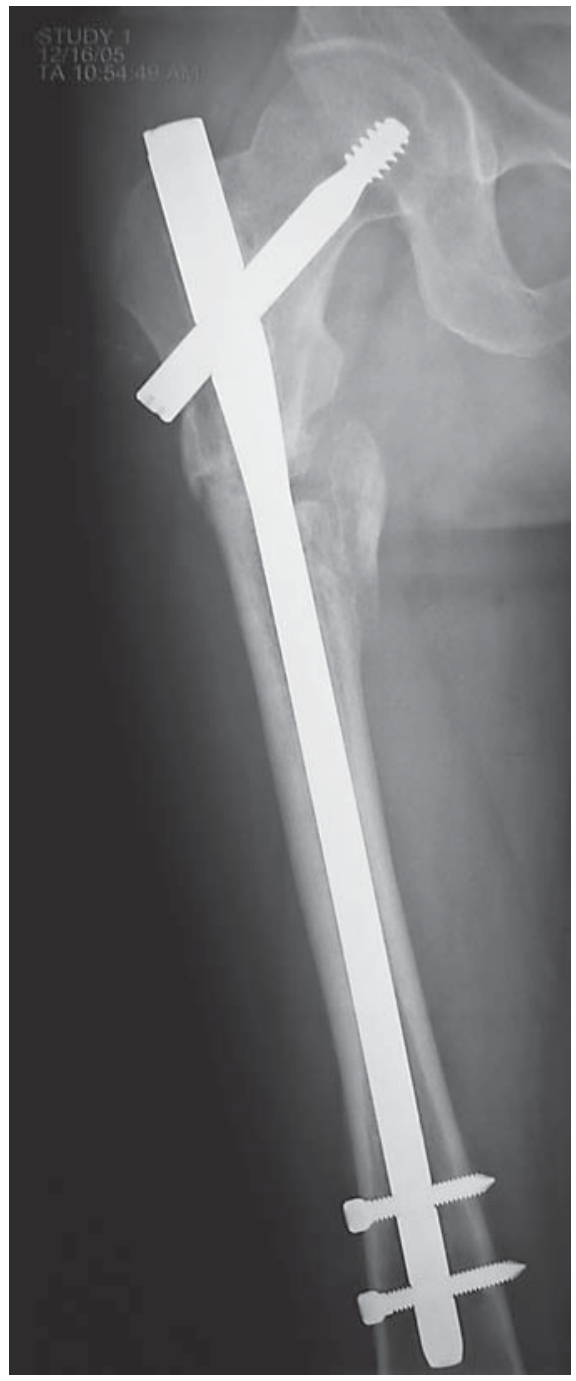

Fig. 3. Fracture nonunion was found 7 months after the first operation.

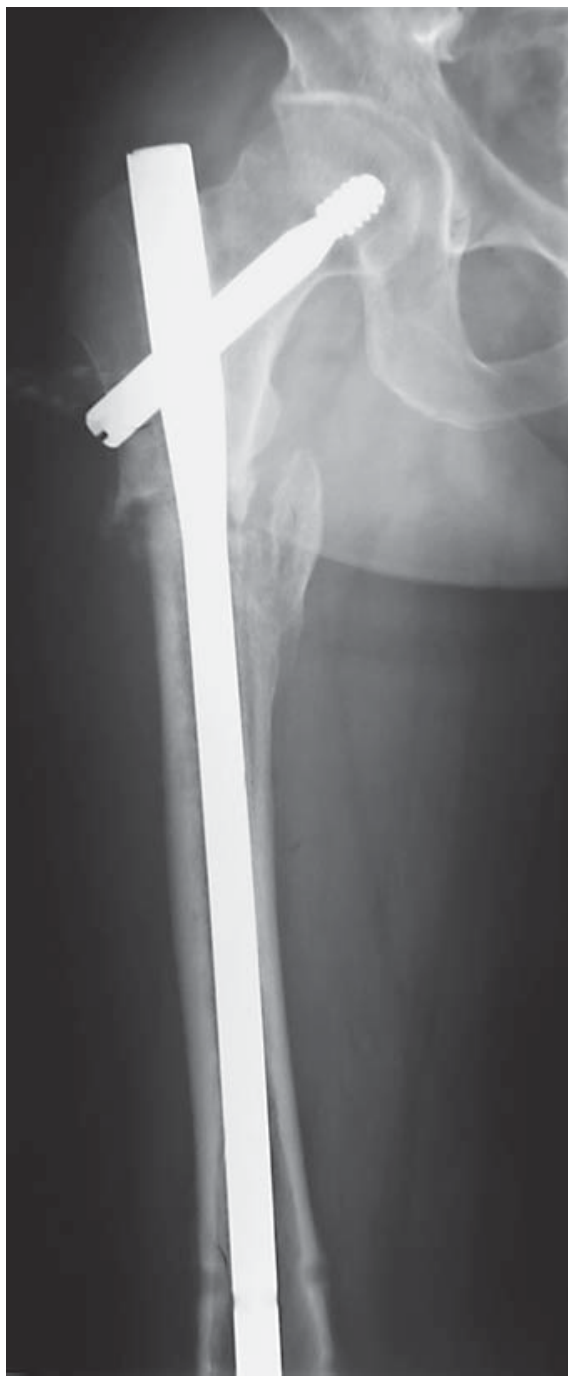

Fig. 4. We removed the two distal locking nails 10 months after the first operation. population, most of the fractures are osteoporotic, resulting from falls [10]. In a survey of subtrochanteric fractures, Velasco and Comfort [3] found that $63 \%$ occurred in patients between 51 and 70 years old and 24\% in patients between 17 and 50 years old. Waddell [4] found a $33 \%$ incidence of subtrochanteric fractures in patients 20-49 years old and a $67 \%$ incidence in patients between 50 and 100 years old in their review of proximal femoral fractures. Young patients accounted for more cases in our study, which may be attributable to the high incidence of car accidents in this region. In contrast to the low-energy injuries in older adults, patients with high-energy injuries often also have comminuted fractures and apparent dis- placement and often show type III, type IV, or even type $\mathrm{V}$ subtrochanteric fractures. If the fracture is not well reconstructed, it will lead to complications, such as implant failure, nonunion, and coxa vara. Some researchers have suggested that when a closed reduction cannot restore good alignment, then open reduction should be undertaken to rebuild the anatomy [11].

Unlike the intertrochanteric region, the subtrochanteric region is composed of hard, cortical bone. The medial and lateral sides routinely suffer strong compressive stresses and tensile stresses, respectively. In the early 20th century, Koch [12] analyzed proximal femur biomechanics during weight-bearing. He showed that up to 1,200 lb/ 


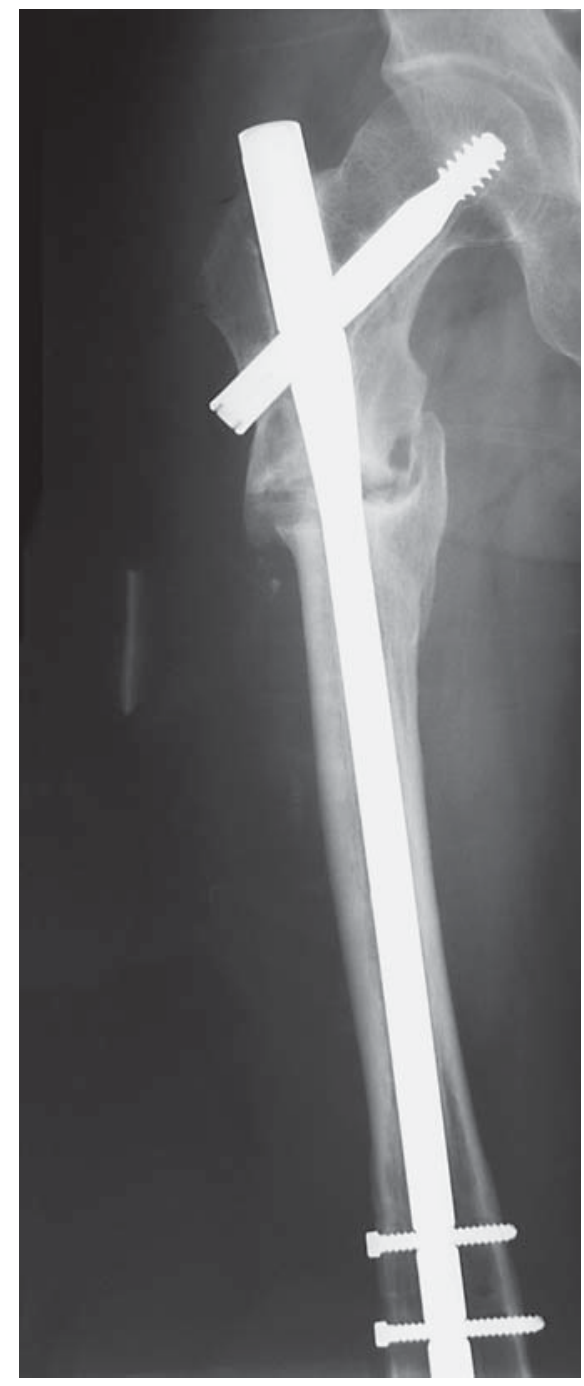

Fig. 5. At 13 months after removing the distal locking nails, the fracture still had not united. Loosening of the Gamma nail can be seen. The distal locking nails were reinserted.

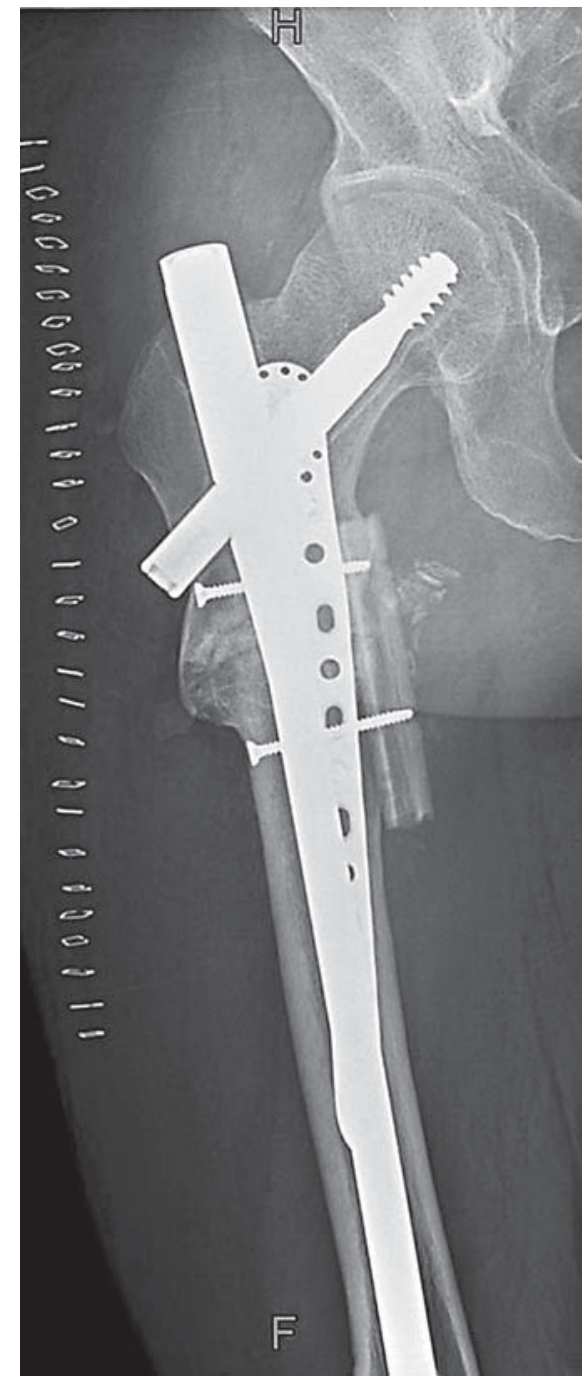

Fig. 6. At 20 months after reinserting the distal locking nails, the fracture still had not united. We used a free fibular transplantation on the medial side and a locking plate on the anterior side.

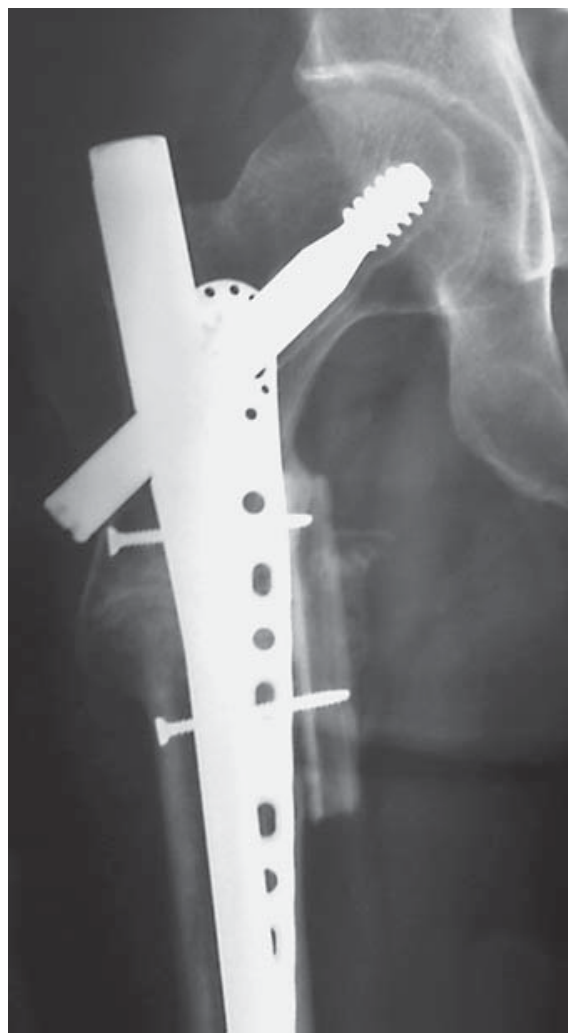

Fig. 7. At 6 months after the fibular transplantation, callus formation was found at the fracture site. in $^{2}$ of force can be generated by a $200-1 b$ man. Compression stress exceeds $1,200 \mathrm{lb} / \mathrm{in}^{2}$ in the medial subtrochanteric area 1-3 in below the level of the lesser trochanter. Lateral tensile stresses are approximately $900 \mathrm{lb} / \mathrm{in}^{2}[12$, 13]. This analysis took into account the additional effects of muscle force. Because of the muscle strength around the hip joint, the proximal femur still suffers considerable stress even when the patient is on bed rest, according to Frankel and Burstein [14].

Presently, the routine surgical treatments for subtrochanteric fractures are intramedullary and extramedul- lary fixation. Although there are reports of such treatment achieving good results, there are also reports of failure. Celebi et al. [15] reported 33 cases of comminuted subtrochanteric fracture treated with dynamic condylar screws and condylar blade plates. In all cases, union was achieved after an average of 15.1 weeks of follow-up. There was no case of delayed union or nonunion [15]. Sourav et al. [16] also reported a series of 102 traumatic subtrochanteric fractures treated with intramedullary nailing. Although there were some cases of malunion and nonunion, it was suggested that this was primarily caused 
by bad reduction and hip varus. The researchers believed that intramedullary nailing in the treatment of subtrochanteric fractures could achieve a $95 \%$ rate of healing [16]. Chang et al. [17] reported that in some cases with marrow cavity narrowing or comminuted entry points that are not suitable for intramedullary nailing, use of minimally invasive techniques and locking compression plates could result in satisfactory results.

Fracture comminution, choice of fixation, excessive soft tissue stripping and incorrect operation technique may be reasons for failure [5]. However, a factor that should not be ignored is bad fracture reduction, especially with no buttress for the medial cortex. Fielding et al. [18] in a 1974 analysis of the biomechanics of subtrochanteric fractures showed that a medial cortical buttress was required to minimize local stress. They noted that nonunion inevitably resulted in fatigue failure of a fixation device and that nonunion was, in fact, the cause of implant failure [18]. When the integrity of the medial cortex is maintained, a lateral fixation device can act as a tension band. When there is a defect in the medial cortex, lateral fixation will be subjected to high bending stresses. This will likely result in the loosening of internal fixation or implant failure, leading to nonunion. In our study, 1 case of type IIIA subtrochanteric fracture was fixed with a long Gamma nail, and we neglected the medially displaced fracture reduction. Nonunion became apparent 7 months after the operation. Removing the distal locking nails and reinserting the locking nails did not work. Then we used a free fibular transplantation on the medial side and a locking plate on the anterior side 43 months after the first operation. Fracture union was achieved 6 months after this revision surgery. This case shows that an internal fixation device was not sufficient to provide adequate stability for a medial cortex defect. The existence of ex- cessive movement at the fracture site will cause nonunion and even implant failure. In other cases in our study for which we used wire or titanium cable cerclage, bone union was achieved without delayed union or implant failure. Qiu and Liu [19] reported a series of 7 subtrochanteric fracture cases treated with proximal femoral nail fixation and titanium cable cerclage. All cases showed union, at an average of 16 weeks; no cases of implant failure, infection, or coxa vara occurred [19].

We do not recommend overstripping soft tissue to achieve anatomical reduction of the medial cortex. Studies have shown that in reducing the medial posterior cortex, stripping too much periosteal tissue will lead to nonunion [20]. If a closed reduction achieves sufficient alignment of the fracture to protect blood supply and promote bone healing, then an open reduction and internal fixation of a medial fracture are not necessary. But if the medial cortex is apparently displaced, it should be reduced and fixed. Otherwise, it may result in nonunion or failure of internal fixation.

\section{Conclusion}

It is regretful that there is no control group to make this retrospective study more scientific. At the same time, there was only 1 typical case in which the medial cortex was not reduced in this series. It was not enough to confirm the hypothesis that bone healing cannot be achieved without medial cortex reduction. Like the other factors such as fracture type, choice of fixation, excessive soft tissue stripping and incorrect operation techniques, medial cortex reduction was only one of the factors we should take into consideration in treating subtrochanteric fractures.

\section{References}

1 Bergman GD, Winquist RA, Mayo KA, Hansen ST: Subtrochanteric fracture of the femur: fixation using the Zickel nail. J Bone Joint Surg Am 1987;69:1032-1040.

2 Boyd HB, Griffin LL: Classification and treatment of trochanteric fractures. Arch Surg 1949;58:853-866.

-3 Velasco RU, Comfort TH: Analysis of treatment problems in subtrochanteric fractures of the femur. J Trauma 1978;18:513-522.

4 Waddell JP: Subtrochanteric fractures of the femur: a review of 130 patients. J Trauma 1979; 19:582-592.

5 Bedi A, Toan Le T: Subtrochanteric femur fractures. Orthop Clin North Am 2004;35: 473-483.
6 Wu CC: Locked nailing for shortened subtrochanteric nonunions. Clin Orthop Relat Res 2009;467:254-259.

7 Nobuyuki Y, Yoshinobu W, Nobuyuki T, Nobuyoshi W, Yukihisa F, Nobuhiko F, Norio M, Hideshi S, Shinro T: Implant failure of long Gamma nail in a patient with intertrochanteric-subtrochanteric fracture. J Orthop Sci 2006;11:638-643.

$\checkmark 8$ Wilhelmina E, Gunnar N, Eva S, Nils D, Jan T: Quality of life after a subtrochanteric fracture: a prospective cohort study on 87 elderly patients. Injury 2009;40:371-376.

$\checkmark 9$ Seinsheimer F: Subtrochanteric fractures of the femur. J Bone Joint Surg Am 1978;60: 300-306. 
$>10$ Trafton PG: Subtrochanteric-intertrochanteric femoral fractures. Orthop Clin North Am 1987;18:59-71.

11 Gibbons CL, Gregg-Smith SJ, Carrell TW, Murray DW, Simpson AH: Use of the Russell Taylor reconstruction nail in femoral shaft fractures. Injury 1995;26:389-392.

12 Koch JC: The laws of bone architecture. Am J Anat 1917;21:177-298.

13 Tordis TG: Stress analysis of the femur. J Biomech 1969;2:163-174.

14 Frankel VH, Burstein AH: Orthopaedic Biomechanics. Philadelphia, Lea \& Febiger, 1970.
15 Celebi L, Can M, Muratli H, Yagmurlu MF, Yuksel HY, Bicimoglu A: Indirect reduction and biological internal fixation of comminuted subtrochanteric fractures of the femur. Injury 2006;37:740-750.

16 Sourav S, Phillip J, Ahmad MA, Henry WJ, Patel AD, Walton NP: Outcome of traumatic subtrochanteric femoral fractures fixed using cephalo-medullary nails. Injury 2007;38: 1286-1293.

17 Chang WO, Jung JK, Young SB, Jong KO, Joon WK, Shin YK, Byung CP, Hyun JL: Minimally invasive plate osteosynthesis of subtrochanteric femur fractures with a locking plate: a prospective series of 20 fractures. Arch Orthop Trauma Surg 2009;129:16591665.
8 Fielding JW, Cochran GV, Zickel RE: Biomechanical characteristics and surgical management of subtrochanteric fractures. Orthop Clin North Am 1974;5:629-650.

19 Qiu HB, Liu ML: Proximal femur nailing and titanium cerclage to treat subtrochanteric fractures. Chin J Bone Joint Injury 2008;23: 153-154.

20 Kinast C, Bolhofner BR, Mast JW, Ganz R: Subtrochanteric fractures of the femur: results of treatment with the 95 degree condylar blade plate. Clin Orthop 1989;238:122130 